\title{
Feasibility of Employing Model-Based Optimization of Pulse Amplitude and Electrode Distance for Effective Tumor Electropermeabilization
}

\author{
Davorka Šel, Alenka Maček Lebar, and Damijan Miklavčič*
}

\begin{abstract}
In electrochemotherapy (ECT) electropermeabilization, parameters (pulse amplitude, electrode setup) need to be customized in order to expose the whole tumor to electric field intensities above permeabilizing threshold to achieve effective ECT. In this paper, we present a model-based optimization approach toward determination of optimal electropermeabilization parameters for effective ECT. The optimization is carried out by minimizing the difference between the permeabilization threshold and electric field intensities computed by finite element model in selected points of tumor. We examined the feasibility of model-based optimization of electropermeabilization parameters on a model geometry generated from computer tomography images, representing brain tissue with tumor. Continuous parameter subject to optimization was pulse amplitude. The distance between electrode pairs was optimized as a discrete parameter. Optimization also considered the pulse generator constraints on voltage and current. During optimization the two constraints were reached preventing the exposure of the entire volume of the tumor to electric field intensities above permeabilizing threshold. However, despite the fact that with the particular needle array holder and pulse generator the entire volume of the tumor was not permeabilized, the maximal extent of permeabilization for the particular case (electrodes, tissue) was determined with the proposed approach.

Model-based optimization approach could also be used for electro-gene transfer, where electric field intensities should be distributed between permeabilizing threshold and irreversible threshold-the latter causing tissue necrosis. This can be obtained by adding constraints on maximum electric field intensity in optimization procedure.
\end{abstract}

Index Terms-Electrochemotherapy, electro gene transfer, electropermeabilization, modeling, optimization.

\section{INTRODUCTION}

$\mathbf{E}$ LECTROPERMEABILIZATION of cell membranes is a phenomenon which has been in the last few years increasingly exploited for medical purposes. Namely, when

Manuscript received December 30, 2005; revised October 7, 2006. This work was supported in part by the European Commission, within the 5th framework programme under the grant Cliniporator QLK3-1999-00484 and by Slovenian Research Agency. Asterisk indicates corresponding author.

D. Šel and A. M. Lebar are with the University of Ljubljana, Faculty of Electrical Engineering, Tržaška 25, SI-1000 Ljubljana, Slovenia (e-mail: davorka.sel@lbk.fe.uni-lj.si; alenka.maceklebar@lbk.fe.uni-lj.si).

D. Miklavčič is with the University of Ljubljana, Faculty of Electrical Engineering, Tržaška 25, SI-1000 Ljubljana, Slovenia (e-mail: damijan.miklavcic@lbk.fe.uni-lj.si).

Color versions of one or more of the figures in this paper are available online at http://ieeexplore.ieee.org.

Digital Object Identifier 10.1109/TBME.2006.889196 exposing cells to the appropriate, short, intense electric pulses the reversible change of their membrane permeability appears [1]-[3], enabling the transfer of molecules and even macromolecules which otherwise cannot enter the cell, such as proteins, some drugs, and nucleic acids.

This phenomenon is already exploited for clinical purposes in order to facilitate the transfer of chemotherapeutics into cells [4]-[12]. The corresponding therapeutic method is referred to as electrochemotherapy [13]. In preclinical trials, another application is currently taking place, where DNA is transferred into cells by means of an applied external electric field [14]-[25]. The method is termed as electrogentransfection and is currently gaining a lot of attention, because it is considered a safer method compared to other methods of gene transfer using viral vectors [26].

As electric field (E) distribution in tissue can be controlled by the applied pulse amplitude and electrode design [27]-[29], determination of optimal electropermeabilization parameters (EP) is crucial for effective tissue permeabilization. In the case of ECT, effective electropermeabilization is achieved when the entire volume of the tumor is exposed to $\mathrm{E}$ intensities above reversible threshold, while in electrogenetransfer, for effective permeabilization, the subjected tissue should be exposed to E intensities distributed between reversible and irreversible threshold. The latter meaning the threshold where tissue necrosis appears. The determination of optimal EP parameters (amplitude, electrode design) should, therefore, consider the requirements of the particular application of permeabilization [27], [29]-[31].

There are different approaches towards optimization of EP parameters. Usually, they are based on information of in vitro [32] and in vivo [28], [33] tests used to determine the E intensity needed for tissue permeabilization. Model-based approaches to optimization of EP parameters, however, have not been used so far, due to the lack of appropriate models describing tissue permeabilization. Namely, the models which have been developed to describe tissue permeabilization [29], [31], [34], did not incorporate changes of tissue permeability due to exposure to external E, i.e., $\sigma(\mathrm{E})$ dependency. Recently presented model of tissue permeabilization [35], [36], however, incorporates $\sigma(\mathrm{E})$ dependency and as such introduces basis for model-based optimization of EP parameters.

In general, parameters subject to optimization could be pulse parameters (shape, amplitude, frequency, pulse duration) [37] and electrode parameters (shape, position and also diameter in case of needle electrodes). Model-based optimization of all the 
stated parameters would require a permeabilization model comprising all listed parameters and also tissue parameters. The development of such a model on a tissue level is too complex and probably would result in a model describing only a narrow range of parameter values.

Within our present work we, thus, narrowed the problem to the optimization of two parameters only: the pulse amplitude $u$ and the distance between electrodes $d$. We investigated the feasibility of model-based optimization of EP parameters on the sequential model of tissue permeabilization presented in previous work of our group [35], [36]. Similarly, optimization on the cell level could have been performed on the pharmacokinetics-based model [38], which is taking into account the pulse duration and pulse amplitude.

At the beginning of the paper the model geometry generation is described, which is derived from computer tomography (CT) images of patient with brain tumor. Then the optimization problem in electrochemotherapy is defined. The model-based optimizations are carried out by considering pulse amplitude and distance between the electrodes (where applicable) as EP parameters subject to optimization. The goal of the optimization was to permeabilize the whole tumor, i.e., to expose the entire volume of the tumor to E intensity values above reversible threshold as used in ECT, assuming this leads to $100 \%$ effectiveness of ECT. Finally the advantages and disadvantages of the model-based approach are described, alleging that the approach can be effectively used for ECT as well as for electro-gene transfer treatment planning.

\section{MATERIALS AND METHODS}

\section{A. Model Geometry, Electrodes, Pulse Generator}

The feasibility study of model-based optimization was carried out on the model geometry representing the brain tissue with tumor. The reason for choosing such geometry was the availability of CT images needed for automatic model geometry generation and the fact that brain tissue can very well illustrate how important is not to damage too much the surrounding tissue by ECT, but just the tumor. In addition, since the chosen geometry is quite complex it can better illustrate the advantages and disadvantages of the approach caused by the geometry and finite element method used. By all means the procedures described could be applied to other types of tissues and locations as well as for other medical image modalities.

For ECT application an array of needle electrodes was used. The needle array, which corresponded to the Cliniporator needle holder, was selected such as to produce the electric field distribution to cover the whole tumor. The Cliniporator TM (IGEA srl, Carpi, Italy) is an electroporation medical device, which was used to deliver high voltage electroporative pulses. The Cliniporator was developed within the Cliniporator project, supported by the European Commission within the 5th framework programme (grant QLK3-1999-00484). Cliniporator needle holder has place for up to 4 pairs of needle electrodes of $0.7 \mathrm{~mm}$ diameter. The distance between the two rows is $8.7 \mathrm{~mm}$ (centers of needle insertion), while the distance between the centers of neighboring needles in the row is $3.2 \mathrm{~mm}$. In clinics, the use of

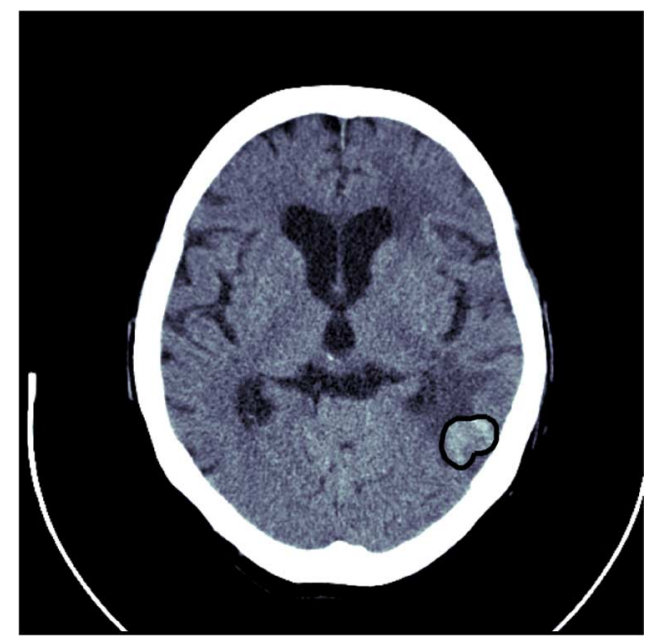

Fig. 1. Head CT image with manually selected polygonal region on the top (black line), which defines the edge of the tumor.

needle electrodes for brain tumor ECT would be carefully considered in order to decide weather it is appropriate for use or not. Thus, the selected geometry should be considered solely as an example to examine the feasibility of the proposed model-based optimization.

The first step of the feasibility study was to develop the model geometry to be used to compute electric field distribution in tissue by means of finite element method (FEM). Thus, the details of the model geometry - the brain, tumor and needle array are given in the following sections together with the explanation of the process of automatic model generation from CT images.

1) Automatic 3-D Geometry Model Generation From CT Images: A substantial part of the modeling effort was put into the generation of brain model geometry from CT images. Head CT images of a patient with a brain tumor were scanned at the Institute of Oncology, Ljubljana, Slovenia. The head scan consisted of 22 slices, each $5 \mathrm{~mm}$ thick. The imaging plane was rotated by $24^{\circ}$ with respect to the horizontal plane (tilt: $-24^{\circ}$ ). The CT images were stored in Digital Imaging and Communications in Medicine (DICOM) file format.

For the purpose of 3-D brain geometry reconstruction from CT brain images, we used every second slice to avoid generating too complex geometry for subsequent mesh generation.

Fig. 1 presents one of the original CT images obtained from the Institute of Oncology. As seen, the bone (skull) is represented in white, while the liquid and the air are represented in black. Considering the wide range of attenuation values, it is hard to expect to differentiate between the white and the grey matter, both shown in shades of grey in Fig. 1. Thus, we decided to model the brain as a homogenous tissue for the purpose of 3-D brain geometry model generation as well as to model the brain tumor as a homogeneous tissue.

We performed CT brain image processing using Matlab (MathWorks Inc., Natick, MA) software and 3-D geometry generation from processed images with Femlab software (Comsol AB, Stockholm, Sweden). Our goal also was to design a process of 3-D model generation which requires the least amount of human interaction possible. The first step in 3-D brain geometry model generation was to determine the edge of 

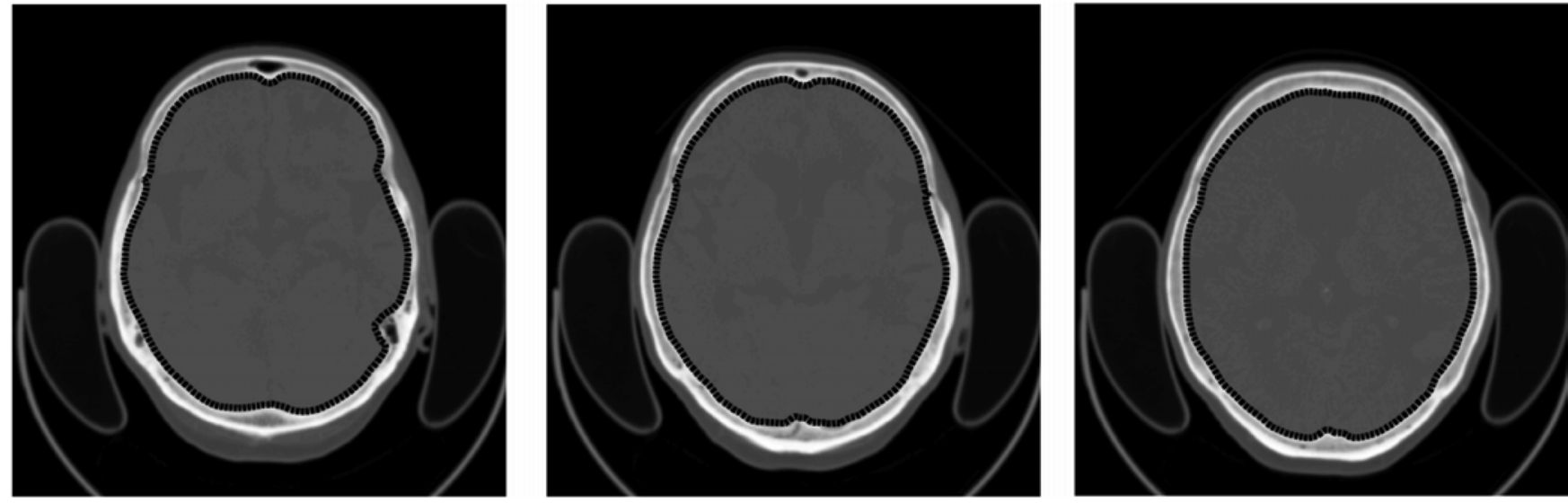

Fig. 2. Brain edge contour curves (black dashed line) on the top of imported CT images-example for 3 slices.

the brain at each CT image (Fig. 2) by defining threshold value and using a contour function, which creates contour curves of the image at a predefined threshold value. Then, 3-D geometry model generation was performed from obtained contour curves with Femlab software by using cubic lofting [39]. The described process of 3-D brain geometry model generation is almost fully automatic. From the user it requires only to set the greyscale threshold value of the brain edge, which is needed for the generation of brain contour curve.

The CT images under our study had low spatial and low contrast resolution, which were the reasons preventing unambiguous automatic differentiation of the tumor from the rest of the brain. Consequently, the 3-D geometry model generation described above for brain required modification for tumor derivation. Instead of automatically determining the edge of the tumor, we manually selected a polygonal region just on the edge of the tumor on the enlarged part of the CT image and in this manner acquired the coordinates for the edge of the tumor. The selected polygonal region is superimposed on the CT scan in Fig. 1. Due to the fact that the tumor was visible in the fourth through seventh CT image, we used each of these images for the 3-D tumor geometry model generation, not only every second as in the case of the 3-D brain geometry model generation. The rest of the 3-D tumor geometry model generation was the same as with the brain. Fig. 3 presents the $x y$ projection of the combined geometry (brain, tumor, and skull). The skull geometry was obtained in the same way as brain geometry from CT images of the patient head.

We inserted the needle electrode array in the brain geometry through the area where the tumor was closest to the edge of the brain. We also took care when inserting needle electrodes that the resulting E distribution will cover the greatest area of tumor possible. The depth of needle penetration $(14 \mathrm{~mm})$ was chosen not to exceed the dimension of the tumor in the direction towards the centre of the brain, which would otherwise damage healthy brain tissue. Fig. 4(b) presents the close up of the position of the needle array with respect to the brain and tumor. We can see that the tumor has a very irregular shape and that the tumor is close to the edge of the brain.

The described geometry consisted of brain, tumor and needles is demanding for automatic mesh generation as it is composed of

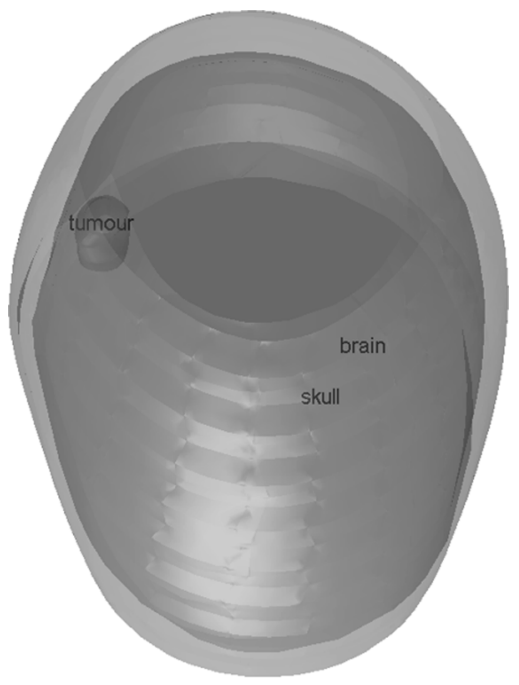

Fig. 3. Generated 3-D geometry model of the skull, brain, and tumor-projection in the $x y$ plane.

irregularly curved objects, which significantly differ in dimensions and have very small distance between the boundaries of two curved objects. Even though employing automatic meshing, the FEM mesh generation was a long process subject to initial mesh parameter tuning. The obtained mesh which consists of 39298 elements is presented in Fig. 4(a). Finer mesh could be used, but was not investigated because finer mesh would significantly increase time and computer resources needed for subsequent optimization, while not contributing to the feasibility study.

\section{B. Model Based Optimization}

Optimization is based on the sequential model of tissue permeabilization presented and described in our previous work [36], which is for the time being the only relevant model describing the change in tissue conductivity due to permeabilization. Parameters subject to optimization were pulse amplitude $u$ and electrode distance $d$. 

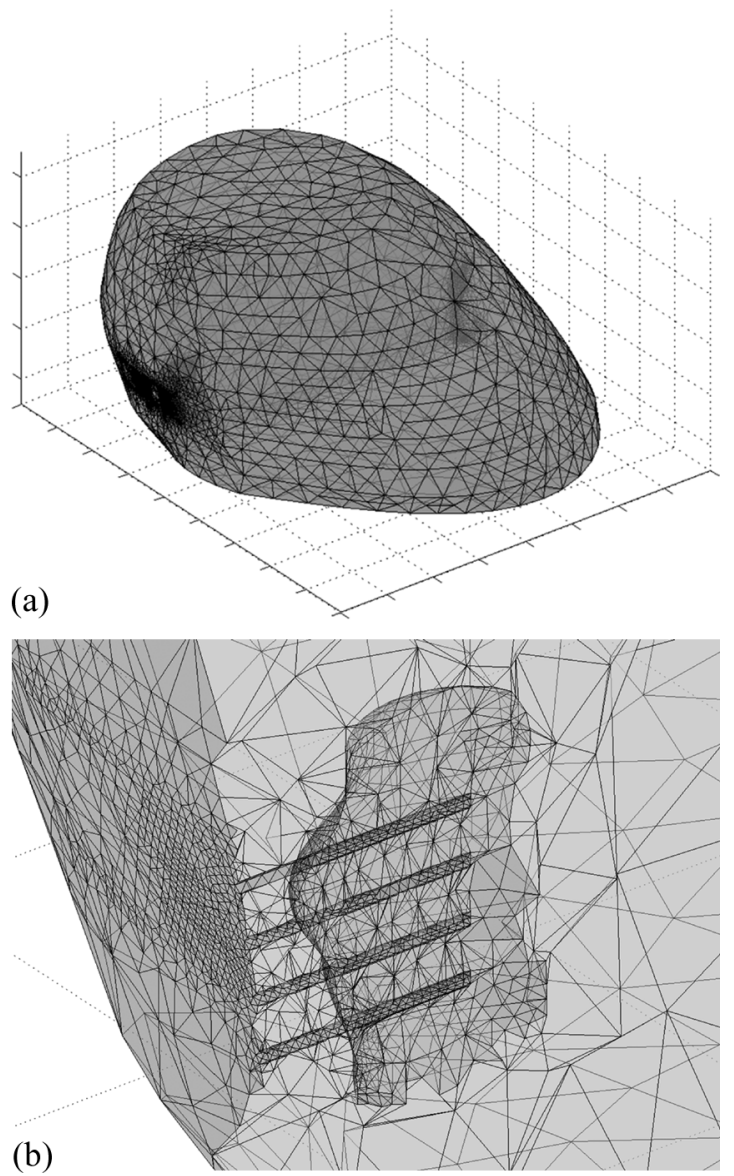

Fig. 4. Finite element mesh of the brain with a tumor and inserted needle electrode array: (a) 3-D perspective of whole geometry; (b) 3-D close up of tumor in cross section parallel to needle length.

Considering the two parameters the objective function has the following form:

$$
\min _{u, d} f\left(E_{r}, E(u, d)\right)
$$

where $E_{r}$ denotes reference value to be reached by optimization and $E(u, d)$ actual value of electric field obtained by applying pulse amplitude $u$ at electrodes distanced at $d$. The objective function to be minimized is defined with Theil's criteria [40]

$$
f\left(E_{r}, E(u, d)\right)=\frac{\sqrt{\sum_{j}\left(E_{r}-E_{j}(u, d)\right)^{2}}}{\sqrt{\sum_{j} E_{r}^{2}}+\sqrt{\sum_{j} E_{j}^{2}(u, d)}}
$$

where $E_{j}$ represents $\mathrm{E}$ intensity computed by the model at $j$ th point in the tumor. For assessment of the objective function, E was computed in five points at the end of permeabilization propagation: $E_{1}\left(x_{1}=60.34 \mathrm{~mm}, y_{1}=174.4 \mathrm{~mm}, z_{1}=30 \mathrm{~mm}\right)$, $E_{2}\left(x_{2}=54.55 \mathrm{~mm}, y_{2}=170.7 \mathrm{~mm}, z_{2}=34 \mathrm{~mm}\right)$, $E_{3}\left(x_{3}=55.26 \mathrm{~mm}, y_{3}=167 \mathrm{~mm}, z_{3}=11.06 \mathrm{~mm}\right)$, $E_{4}\left(x_{4}=53 \mathrm{~mm}, y_{4}=158.5 \mathrm{~mm}, z_{4}=20 \mathrm{~mm}\right)$, and $E_{5}\left(x_{5}=54.55 \mathrm{~mm}, y_{5}=159 \mathrm{~mm}, z_{5}=15 \mathrm{~mm}\right)$. They are critical as they represent points on the edge of the tumor where the lowest $\mathrm{E}$ intensities are expected due to electrode positioning. These points are shown in Fig. 5(a), (c), and (e). E could have been evaluated in all points of the tumor but we have chosen only critical points in order to hasten time needed for optimization. The reference value of $\mathrm{E}$ to be reached in each of those points was $E_{r}=255 \mathrm{~V} / \mathrm{cm}$, which was defined slightly above tumor tissue reversible threshold $E_{0 t}=250 \mathrm{~V} / \mathrm{cm}$ in order to assure tumor permeabilization. The reversible threshold of tumor tissue was defined hypothetically, for the reason further explained in the Tissue Parameters chapter.

Taking into account the technical limitations of the Cliniporator, which supplies limited voltage $u_{\max }=1000 \mathrm{~V}$ and current $i_{\max }=16 \mathrm{~A}$, we defined constraints on voltage $(u)$, subject to optimization

$$
0<u<u_{\max }
$$

and we set a limitation on current as a nonlinear constraint of the following form:

$$
c_{1}(u, d)=\left(i(u, d)-i_{\max }\right) \leq 0
$$

where $i(u, d)$ denotes the total current computed by the model. In addition, our goal was to ensure that $E$ in predetermined point $j$ of the tumor exceeds reversible threshold. Thus, we set additional nonlinear constraints for each evaluation point $j$

$$
c_{2 j}(u, d)=\left(E_{r}-E_{j}(u, d)\right) \leq 0 .
$$

Constraints on the second optimization parameter-distance $d$ were set considering the characteristic of the model geometry and by considering the electrode holder dimensions. Thus, they will be described later in this paper.

\section{Tissue Parameters}

With respect to the final goal, i.e., to optimize EP parameters for electrochemotherapy of a brain tumor based on real geometry extracted from CT images, we used tissue electric parameters which correspond to brain and tumor tissue.

The conductivity $(\sigma)$ of brain tissue reported in literature for animals (cow, pig, and rabbit) at low frequency and at body temperature was $0.17 \mathrm{~S} / \mathrm{m}$, with grey matter conductivity 0.35 $\mathrm{S} / \mathrm{m}$ and white matter $0.15 \mathrm{~S} / \mathrm{m}$ [41]. The same value, i.e., $0.17 \mathrm{~S} / \mathrm{m}$ was reported for average human brain conductivity in [42], while the measurement at $1 \mathrm{kHz}$ reported in [43] estimated human brain conductivity in the range between $0.20-0.22 \mathrm{~S} / \mathrm{m}$. Considering a possible increase in conductivity at $1 \mathrm{kHz}$, we used the lower value of human brain conductivity $\left(\sigma_{0 \mathrm{~b}}=0.17 \mathrm{~S} / \mathrm{m}\right)$ in this work.

There were no reported values of brain tumor conductivity in literature available to us. Thus, we based the value of brain tumor conductivity on the fact, that tumor tissue has in general higher conductivity than the surrounding tissue [44]-[46]. Considering this in all subsequent models we have chosen the conductivity of brain tumor tissue to be 2.2 times larger than the conductivity of nonpermeabilized brain tissue, which corresponds 

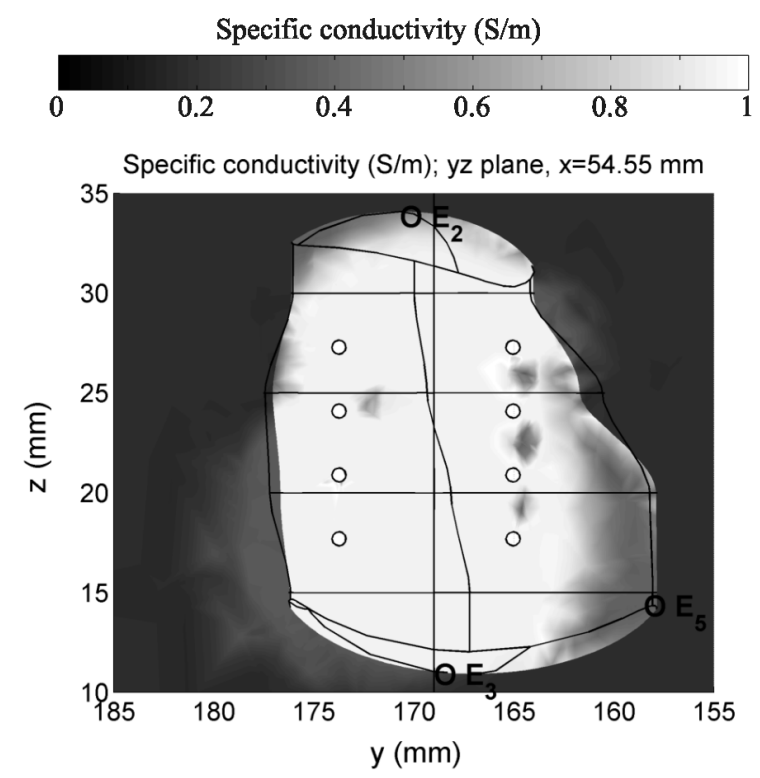

(a)

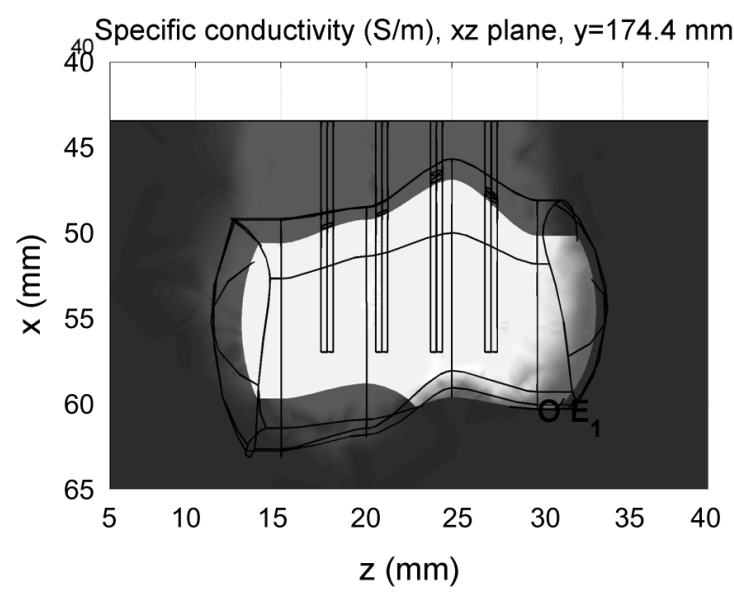

(c)

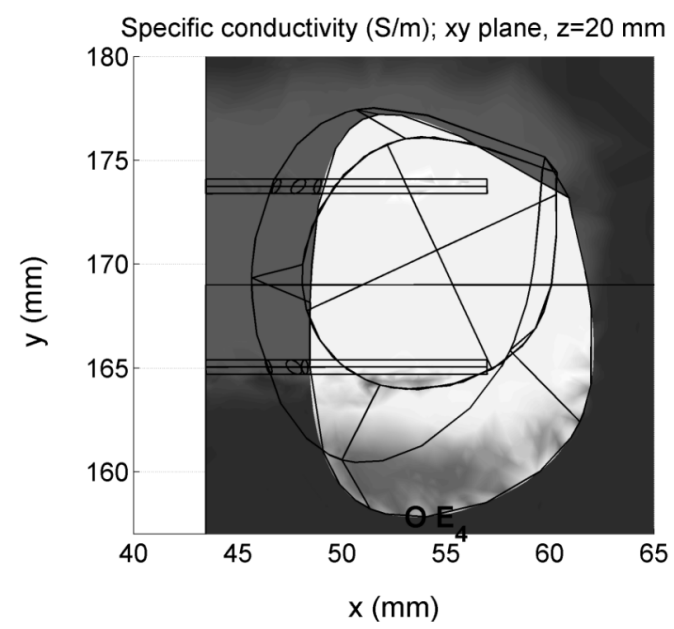

(e)

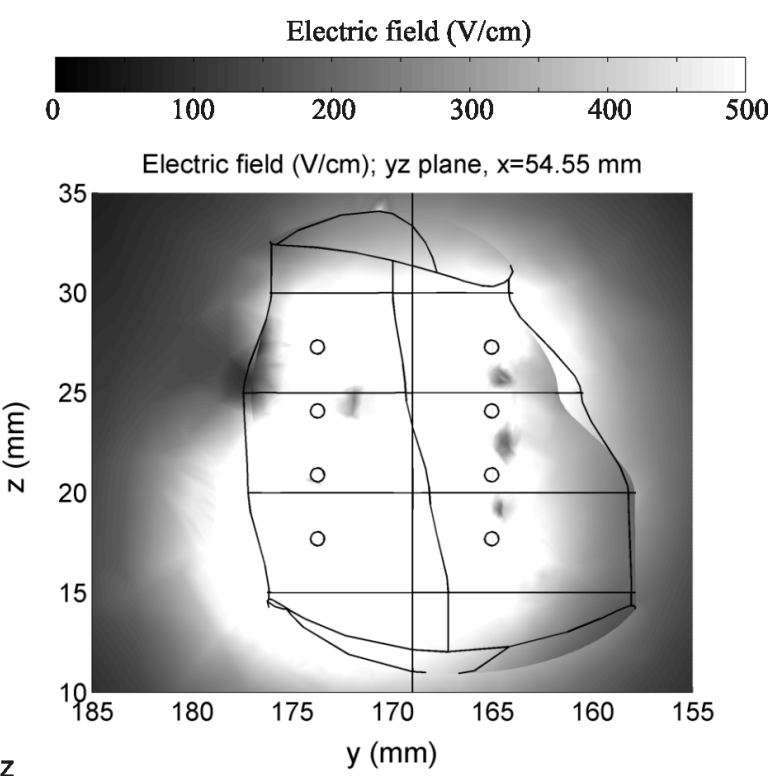

(b)

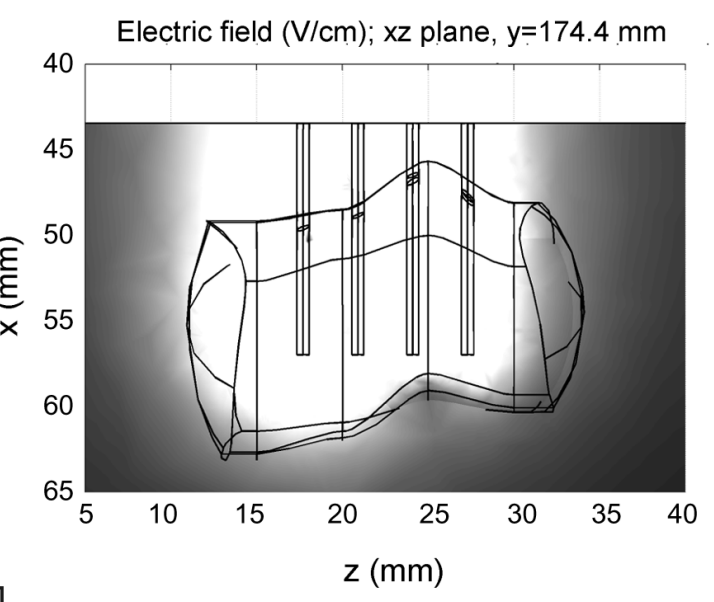

(d)

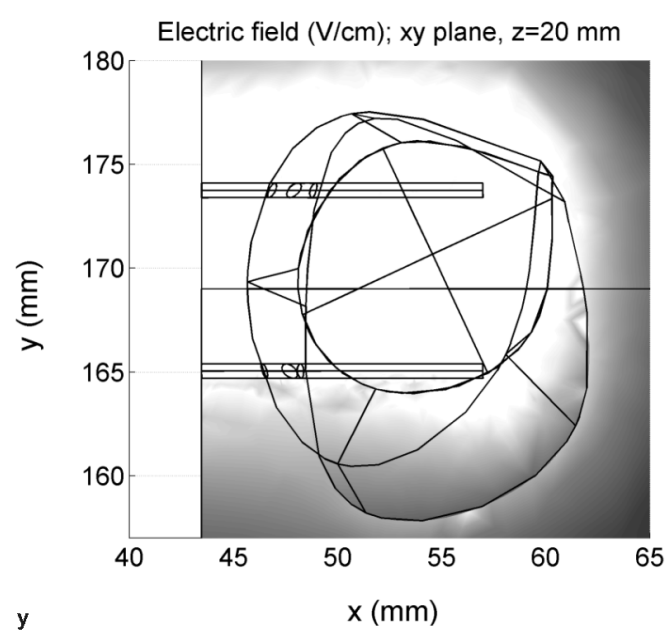

(f)

Fig. 5. Conductivity in $x y, x z$ and $x y$ planes shown in (a), (c), and (e), respectively, and corresponding electric field at the end of permeabilization in $x y$, $x z$ and $x y$ planes shown in (b), (d), and (f), respectively. Results are given for model 5.

to $\sigma_{0 \mathrm{t}}=0.38 \mathrm{~S} / \mathrm{m}$. This value, however, does not necessarily represent the real brain tumor conductivity and is used only for the purpose of present feasibility study.
The $\sigma(\mathrm{E})$ dependencies of both brain and tumor tissue were approximated with an S-shaped function for the reasons presented in our previous work [36], [47]. Fig. 6 displays both $\sigma(\mathrm{E})$ 


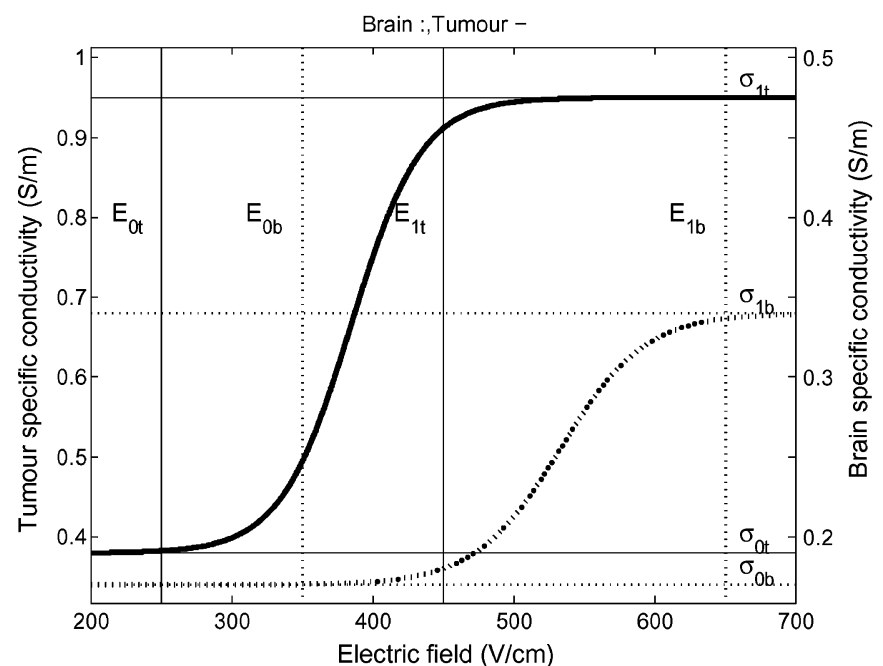

Fig. 6. $\sigma(\mathrm{E})$ dependencies of brain tissue (dashed curve) and tumor tissue (full curve). Note that different scales for brain (right) and tumor (left) tissue conductivity are used.

dependencies of brain and tumor tissue, respectively. The increase in tumor tissue conductivity $\left(\sigma_{1 \mathrm{t}}\right)$ is based on estimated values for a subcutaneous tumor presented in [35]. The rest of the values (brain reversible $\left(\mathrm{E}_{0 \mathrm{~b}}\right)$ and irreversible $\left(\mathrm{E}_{1 \mathrm{~b}}\right)$ thresholds, increased brain tissue conductivity $\left(\sigma_{1 \mathrm{~b}}\right)$, tumor reversible $\left(\mathrm{E}_{0 \mathrm{t}}\right)$ and irreversible $\left(\mathrm{E}_{1 \mathrm{t}}\right)$ thresholds) were defined hypothetically. The hypothetical values were defined to be around the range of known thresholds for other tissue (rabbit liver tissue [36]). The choice of hypothetical values of parameters is not questionable for use in feasibility study, however, for application on real tissue the $\sigma(\mathrm{E})$ dependency should be exactly defined.

\section{RESULTS}

\section{A. Pulse Amplitude and Distance Optimization on Realistic Geometry}

The parameters subject to optimizations were either pulse amplitude $u$ or a combination of $u$ and distance $d$ between two electrode pairs. When incorporating the distance between electrodes as a parameter for optimization we utilized two electrode pairs in an electrode holder instead of four and defined $d$ as the distance between the two electrode pairs. As model geometry changed for different distances the optimization of the distance between electrodes required new mesh generation for each distance. Therefore, we tested mesh generation for different distances between electrodes in advance and found it impossible to build the mesh at certain distances. The reasons were the irregular shape of tumor, small distance between edges of tumor, brain and needles and the huge difference in size between the tissue (tumor size approximately $2 \mathrm{~cm} \times 2.5 \mathrm{~cm} \times 1.5 \mathrm{~cm}$ ) and needles $(\varphi=0.7 \mathrm{~mm})$. Therefore, we transformed the problem of distance optimization into a problem of optimization of discrete distances at which mesh generation succeeded. The transformed optimization problem was required to find the minimum of the objective function subject to one continuous parameter $u$ and one discrete parameter $d$. This is a so called mixed integer programming problem [48], which can be solved either by optimizing continuous parameter at each value of discrete parameter or by using special purpose algorithms, such as a Tree-search algorithm for mixed integer programming problems [49]. We used the first approach, as we tested only two discrete distance values and employed Sequential Quadratic Programming method for constrained nonlinear optimization. However, if several discrete values of $d$ were present we would consider optimization based on the Tree-search algorithm.

We first used the same constraints on $u, i$ and $E_{j}$ as described in (3)-(5) in Section II-B. However, the optimization had no feasible solution as constraints on $u$ and $i$ limited the field distribution $E_{j}$. Thus, we removed the constraint on $E_{j}$ [see (5)) because $u$ and $i$ are physical limitations of the pulse generator and consequently cannot be removed.

Table I presents the value of objective function, optimal pulse parameters, $u$ and $i$ delivered by pulse generator and optimal values of $E_{j}$ at the evaluation points obtained in different models. The model 1 refers to the model with two electrode pairs with the rows at same potential and distance $3.2 \mathrm{~mm}$ between needles in row, similarly model 2 refers to a distance of $9.6 \mathrm{~mm}$ between two electrode pairs, model 3 refers to the model with four needle pairs with rows at the same potential and 4 to the model with different potentials between four needle pairs. Model 5 is similar to model 4 but without constraints on $u$ and $i$. The time needed for the computation of one sequential permeabilization model was $29 \mathrm{~min}$ on the laptop (Intel Pentium III, $1066 \mathrm{MHz}$ CPU, $256 \mathrm{MB}$ RAM). The number of evaluations of objective function within optimizations ranged from 5 evaluations (model 1 and model 2) up to 74 evaluations (model 5). Average time spent on optimization was 12 hours. In Table I, we can see that in model 1 the constraint on u was reached, while in models $2-4$ the constraint reached was on $i$. Non of the models 1-4 did deliver E higher than $E_{r}$ in all selected points. Only model 5, which did not incorporate constraints on $u$ and $i$, provided $\mathrm{E}$ higher than $E_{r}$ in all selected points.

Fig. 5 presents conductivity and E distribution, respectively, of model 5 at the end of permeabilization process. We can observe that increased $\mathrm{E}$ values were concentrated within the tumor which is particularly advantageous because in this way healthy brain tissue is not exposed to higher $\mathrm{E}$ intensities that can cause irreversible brain cell damage. The reason for increased $\mathrm{E}$ values within the tumor is also due to tumor tissue conductivity being higher than brain tissue conductivity, as well as change in tumor conductivity due permeabilization is not higher that change in brain tissue conductivity (see Fig. 5).

Further we established that with the particular needle array holder we cannot permeabilize the entire volume of the tumor with a single needle array insertion considering the constraints on voltage and current supplied by the Cliniporator. However, by employing optimization we can determine pulse amplitude parameters for each electrode pair that can permeabilize the tumor as much as possible considering the constraints. This can consequently decrease the number of needle array insertions and reduce associated tissue damage.

\section{DISCUSSION}

The goal of the model-based optimization was to determine optimal EP parameters to be applied for effective 
TABLE I

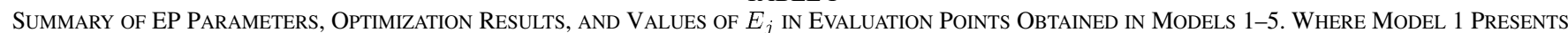
The Model With Two Electrode Pairs at a Distance of $3.2 \mathrm{~mm}$, Model 2 Refers to a Distance of 9.6 mm Between Two Electrode Pairs, Model 3 Refers to the Model With Four NeEdle Pairs With Rows at the SAME Potential and Model 4 to the Model With Different Potentials Between Four Needle Pairs. Model 5 Is the Same as Model 4 But Without Constraints ON $u$ AND $i$

\begin{tabular}{|c|c|c|c|c|c|c|c|c|c|}
\hline Model & $f(u, d)$ & $u(\mathrm{~V})$ & $d(\mathrm{~mm})$ & $i(\mathrm{~A})$ & $\mathrm{E} 1(\mathrm{~V} / \mathrm{cm})$ & $\mathrm{E} 2(\mathrm{~V} / \mathrm{cm})$ & $\mathrm{E} 3(\mathrm{~V} / \mathrm{cm})$ & $\mathrm{E} 4(\mathrm{~V} / \mathrm{cm})$ & $\mathrm{E} 5(\mathrm{~V} / \mathrm{cm})$ \\
\hline 1 & 0.1907 & 1000.0 & 3.2 & 15.07 & 200.3 & 173.6 & 169.3 & 196.6 & 138.6 \\
\hline 2 & 0.1921 & 915.3 & 9.6 & 15.99 & 340.1 & 267.3 & 256.0 & 107.7 & 130.1 \\
\hline 3 & 0.1869 & 701.0 & 1 & 16.00 & 276.9 & 227.5 & 216.7 & 128.6 & 120.8 \\
\hline
\end{tabular}

electrochemotherapy by minimizing the difference between the reference value $\mathrm{Er}$ and $\mathrm{E}$ intensities computed at selected points in the tumor and by considering the requirement that $\mathrm{E}$ intensity at selected points in the tumor should be higher than reversible threshold. Optimization also considered the technical limitations of the pulse generator.

We examined the feasibility of model-based optimization on a model with relatively complex geometry. The model, generated from CT images, represented a human brain with a tumor. For the purpose of electrochemotherapy an array of existing needle electrodes was inserted in the model. The parameter subject to optimization was pulse amplitude. The distance between electrode pairs was optimized as discrete value. During optimizations the constraints on voltage or current were reached, thus, the application of optimal pulse parameters obtained in such a way did not expose the entire volume of the tumor to $\mathrm{E}$ intensities above reversible threshold. Considering the fact that some parts of the tumor were permeabilized while others were not, the treatment should be repeated considering a new position of the electrodes which would permeabilize the areas of the tumor which remained nonpermeabilized by the first needle insertion. Alternatively also other types of electrodes could be used (e.g. hexagonal [50]). Despite the fact that with the particular needle array holder and pulse generator we cannot permeabilize the entire volume of the tumor with a single needle array insertion, we can determine the pulse amplitude to be delivered to each electrode pair separately in order to permeabilize the tumor to a great extent. This can consequently decrease the number of needle array insertions and reduce associated tissue damage. Also, if using the needle electrode holder which enables switching between electrode pairs, the current constraint would probably not prevent permeabilization of the total volume of tissue by optimized pulse amplitudes.

The purpose of the presented feasibility study was to examine the advantages and disadvantages of model-based optimization, which are listed in continuation, as well as to identify possible limitations of the approach.

The most important contribution of model-based optimization of EP parameters is the determination of optimal parameters in a noninvasive way before the treatment. The optimal parameters are also best suited to the treatment of the particular tissue with respect to its geometry and electrical properties.

Further, the optimization enables the determination of optimal pulse amplitude to each electrode pair separately, as well as it could be used for determination of optimal potentials to be applied to 3-D electrodes where different potential can be delivered along the electrode.

Another advantage of the approach is the information about the efficacy of permeabilization, i.e., information whether the entire volume of the tumor would be permeabilized. The latter is derived from the $\mathrm{E}$ distribution computed by the model. This information is extremely valuable when for example a tumor of irregular shape is treated with needle electrodes, which produce highly inhomogeneous $\mathrm{E}$. In such a case, the use of a model providing the spatial distribution of $\mathrm{E}$ is of great importance.

The spatial distribution of $\mathrm{E}$ also provides information about the possible damage to the healthy surrounding tissue when exposed to a particular electrical treatment. Based on this a decision can be made whether to permeabilize a larger area of tissue by applying higher pulse amplitudes or to reposition the electrodes and carry out the treatment under lower pulse amplitudes. The requirement that certain $\mathrm{E}$ intensity should not be exceeded in healthy tissue can also be taken into account within the optimization procedure as a nonlinear constraint.

The model-based optimization can be easily employed in daily clinical work provided there are CT or MR images of the patient available. The model-based optimization is just reusing images, which are primarily taken for diagnostic purposes. The whole model-based optimization process is in fact a simulation, which requires a capable personal computer with special purpose software installed. The whole optimization process does not require much human involvement, but just the choice of electrodes, definition of their position, selection of tissue type and definition of generator constraints. However, the model-based optimization approach must be experimentally validated before the use in clinic and also the software should be further developed to become robust enough for daily use.

For model-based optimization a permeabilization model is required. Thus, the availability of an accurate model could be the major limitation to this approach. The accuracy of the presented time discrete permeabilization model depends predominantly on the quality of the 3-D geometry and determination of $\sigma(\mathrm{E})$ dependency. The former can be generated from CT or MR images if available. The latter should be defined either by means of experiments [35], [36] for each type of tissue separately or theoretically [51].

Another important limitation is optimization of the distance between electrodes when tissue geometry is complex. Each 
change in model geometry, for example a change in the distance between electrodes, requires new FEM mesh generation. When optimizing the distance between electrodes several mesh generations are, thus, required. However, in complex geometries the automatic mesh generation can fail if initial mesh parameters are not manually tuned. To overcome this problem in complex geometries, electrode position and mesh generation should be prepared in advance. Then the discrete values of distances between the electrodes can be optimized together with pulse amplitude by employing the Tree search algorithm, for example. In the case of simple geometries, there should be no problems when optimizing the distance between electrodes.

In general, the definition of the optimization problem in electrochemotherapy should take into consideration also technical limitations of the generator (maximum voltage, current), constraints on electrode dimensions, and the requirement that $\mathrm{E}$ intensity should be higher than the reversible threshold in the entire tumor. However, in some cases a feasible solution to such a problem does not exist. In such cases, the definition of constraints should be redesigned by taking into account only the technical limitations of the generator and electrode dimensions, while the rest of the requirements for desired $\mathrm{E}$ intensity could be met by repositioning the electrodes and repeating the treatment.

\section{CONCLUSION}

Balancing the considerations described in Discussion part we can conclude that model-based optimization can be successfully used as a mean of determining electropermeabilization parameters for effective electrochemotherapy, provided an accurate model of permeabilization is available. However, in some cases the effectiveness may be limited due to the dimensions of modeled tissue, limitations of the pulse generator, and electrode dimensions.

The applicability of this approach should be further validated on the tumor tissue with known tissue parameters which is treated by ECT using computed optimal EP parameters. The volume of permeabilized tissue after permeabilization with optimal pulse amplitude could, for example, be validated by electric impedance tomography which should provide information about conductivity in tissue after permeabilization [52].

In addition to the advantages described in Section IV, it has to be emphasized that the use of model-based optimization could also be advantageous for electrogenetransfer, where optimal pulse amplitude should be determined very precisely in order to have electric field intensities in the target tissue distributed between reversible and irreversible thresholds, thus preserving cell viability. The approach proposed can be also useful for irreversible electroporation—-suggested for tissue ablation [53].

\section{REFERENCES}

[1] T. Y. Tsong, "Electroporation of cell membranes," Biophysical J., vol. 60, pp. 297-306, 1991.

[2] J. C. Weaver and Y. A. Chizmadzhev, "Theory of electroporation: A review," Bioelectrochem. Bioenerg., vol. 41, pp. 135-160, 1996.
[3] E. Neumann and S. Kakorin, "Digression on membrane electroporation and electroporative delivery of drugs and genes," Radiol. Oncol., vol. 32, no. 1, pp. 7-17, 1998 .

[4] M. R. Jaroszeski, R. Gilbert, and R. Heller, "Electrochemotherapy: An emerging drug delivery method for the treatment of cancer," Adv. Drug Delivery Rev., vol. 26, pp. 185-197, 1997.

[5] R. Heller, R. Gilbert, and M. J. Jaroszeski, "Clinical applications of electrochemotherapy," Adv. Drug Delivery Rev., vol. 35, no. 1, pp. 119-129, 1999.

[6] L. M. Mir, "Therapeutical perspectives of in vivo cell electropermeabilization," Bioelectrochemistry, vol. 53, pp. 1-10, 2000.

[7] M. P. Rols, J. M. Bachaud, P. Giraud, C. Chevreau, H. Roche, and J. Teissie, "Electrochemotherapy of cutaneous metastases in malignant melanoma," Melanoma Res., vol. 10, no. 5, pp. 468-474, 2000.

[8] M. Reberšek, T. Čufer, M. Čemažar, S. Kranjc, and G. Serša, "Electrochemotherapy with cisplatin of cutaneous tumor lesions in breast cancer," Anti-Cancer Drugs, vol. 15, pp. 593-597, 2004.

[9] J. P. Allegretti and W. R. Panje, "Electroporation therapy for head and neck cancer including carotid artery involvement," Laryngoscope, vol. 111 , no. 1, pp. 52-56, 2001.

[10] S. Rodriguez-Cuevas, S. Barroso-Bravo, J. Almanza-Estrada, L. Cristobal-Martinez, and E. Gonzalez-Rodriguez, "Electrochemotherapy in primary and metastatic skin tumors: Phase II trial using intralesional bleomycin," Arch. Med. Res., vol. 32, no. 4, pp. 273-276, 2001.

[11] Gothelf, L. M. Mir, and J. Gehl, "Electrochemotherapy: Results of cancer treatment using enhanced delivery of bleomycin by electroporation," Cancer Treatment Rev., vol. 29, no. 5, pp. 371-387, 2003.

[12] G. Serša, M. Čemažar, and Z. Rudolf, "Electrochemotherapy: Advantages and drawbacks in treatment of cancer patients," Cancer Therapy, vol. 1, pp. 133-142, 2003.

[13] L. M. Mir, S. Orlowski, J. Belehradek, and C. Paoletti, "Elecrochemotherapy potentiation of antitumor effect of bleomycin by local electric pulses," Eur. J. Cancer, vol. 27, no. 1, pp. 68-72, 1991.

[14] M. J. Jaroszeski, R. Gilbert, C. Nicolau, and R. Heller, "In vivo gene delivery by electroporation," Adv. Drug Delivery Rev., vol. 35, pp. 131-137, 1999.

[15] M. P. Rols, C. Delteil, M. Golzio, P. Dumond, S. Cros, and J. Teissie, "In vivo electrically mediated protein and gene transfer in murine melanoma," Nature Biotechnol., vol. 16, pp. 168-171, 1998.

[16] H. Aihara and J. Miyazaki, "Gene transfer into muscle by electroporation in vivo," Nature Biotechnol., vol. 16, pp. 867-870, 1998.

[17] L. M. Mir, M. F. Bureau, J. Gehl, Rangara, D. Rouy, J. M. Caillaud, P. Delaere, D. Branellec, B. Schwartz, and D. Scherman, "High-efficieny gene transfer into skeletal muscle mediated by electric pulses," Proc. Nat. Acad. Sci., vol. 96, pp. 4262-4267, 1999.

[18] J. Glasspool-Malone, S. Somiari, J. J. Drabick, and R. W. Malone, "Efficient nonviral cutaneous transfection," Molec. Therapy, vol. 2, no. 2, pp. 140-146, 2000.

[19] S. Satkauskas, M. Breau, M. Puc, A. Mahfoudi, D. Scherman, D. Miklavčič, and L. M. Mir, "Mechanisms of in vitro DNA electrotransfer: Respective contributions of cell electropermeabilization and DNA electrophoresis," Molec. Therapy, vol. 5, no. 2, pp. 1-8, 2002.

[20] M. L. Lucas and R. Heller, "IL-12 gene therapy using an electrically mediated nonviral approach reduces metastatic growth of melanoma," DNA Cell Biol., vol. 22, no. 12, pp. 755-763, 2003.

[21] N. Pavšelj and V. Preat, "DNA electrotransfer into the skin using a combination of one high- and one low-voltage pulse," J. Controlled Release, vol. 106, no. 3, pp. 407-415, 2005.

[22] L. Heller, K. Merkler, and J. Westover, "Evaluation of toxicity following electrically mediated interleukin-12 gene delivery in a B16 mouse melanoma model," Clin. Cancer Res., vol. 12, no. 10, pp. 3177-3183, 2006.

[23] T. Kawano, M. Yamagata, H. Takahashi, Y. Niidome, S. Yamada, Y. Katayama, and T. Niidome, "Stabilizing of plasmid DNA in vivo by PEG-modified cationic gold nanoparticles and the gene expression assisted with electrical pulses," J. Controlled Release, vol. 111, no. 3, pp. 382-389, 2006.

[24] B. M. Medi and J. Sigh, "Skin targeted DNA vaccine delivery using electroporation in rabbits II. Safety," Int. J. Pharmaceutics, vol. 308, no. $1-2$, pp. 61-68, 2006.

[25] A. K. Roos, S. Moreno, C. Leder, M. Pavlenko, A. King, and P. Pisa, "Enhancement of cellular immune response to a prostate cancer DNA vaccine by intradermal electroporation," Molec. Therapy, vol. 13, no. 2, pp. 20-327, 2006. 
[26] D. Ferber, "Gene therapy: Safer and virus-free?," Science, vol. 294, pp. 1638-1642, 2001.

[27] D. Miklavčič, K. Beravs, D. Šemrov, M. Čemazar, F. Demšar, and G. Serša, "The importance of electric field distribution for effective in vivo electroporation of tissues," Biophysical J., vol. 74, pp. 2152-2158, 1998.

[28] J. Gehl, T. H. Sorensen, K. Nielsen, P. Raskmark, S. L. Nielsen, T. Skovsgaard, and L. M. Mir, "In vivo electroporation of skeletal muscle: Threshold, efficacy and relation to electric field distribution," Biochimica et Biophysica Acta, vol. 1428, pp. 233-240, 1999.

[29] K. Brandisnky and I. Daskalov, "Electric field and current distributions in electrochemotherapy," Biochem. Bioenerg., vol. 48, pp. 201-208, 1999.

[30] G. A. Hofmann, S. B. Dev, S. Dimmer, and G. S. Nanda, "Electroporation therapy: A new approach for the treatment of head and neck cancer," IEEE Trans. Biomed. Eng, vol. 46, no. 6, pp. 752-759, Jun. 1999.

[31] D. Miklavčič, D. Šemrov, H. Mekid, and L. M. Mir, "A validated model of in vivo electric field distribution in tissue for electrochemotherapy and for DNA electrotransfer for gene therapy," Biochim. Biophys. Acta, vol. 1523, pp. 73-83, 2000.

[32] M. P. Rols and J. Teissie, "Electropermeabilization of mammalian cells: Quantitative analysis of the phenomenon," Biophysical J., vol. 58, pp. 1089-1098, 1990.

[33] J. Gehl and L. M. Mir, "Determination of optimal parameters for in vivo gene transfer by electroporation, using a rapid in vivo test for cell permeabilisation," Biochem. Biophysical Res. Commun., vol. 261, pp. 377-380, 1999.

[34] S. B. Dev, D. Dhar, and W. Krassowska, "Electric field of a six-needle array electrode used in drug and gene delivery in vivo: Analytical versus numerical solution," IEEE Trans. Biomed. Eng., vol. 50, no. 11, pp. 1296-1300, Nov. 2003.

[35] N. Pavšelj, Z. Bregar, D. Cukjati, D. Batiuskaite, L. M. Mir, and D. Miklavčič, "The course of tissue permeabilization studied on a mathematical model of a subcutaneous tumor in small animals," IEEE Trans. Biomed. Eng., vol. 52, no. 8, pp. 1373-1381, Aug. 2005.

[36] D. Šel, D. Cukjati, D. Batiuskaite, T. Slivnik, L. M. Mir, and D. Miklavčič, "Sequential finite element model of tissue electropermeabilization," IEEE Trans. Biomed. Eng., vol. 52, no. 5, pp. 816-827, May 2005.

[37] S. Satkauskas, D. Batiuskaite, S. Salomskaite-Davalgiene, and M. S. Venslauskas, "Effectiveness pf tumour electrochemotherapy as a function of electric pulse strength and duration," Bioelectrochemistry, vol. 65, pp. 105-111, 2005.

[38] M. Puc, T. Kotnik, L. M. Mir, and D. Miklavčič, "Quantitative model of small molecules uptake after in vitro cell electropermeabilisation," Bioelectrochemistry, vol. 60, pp. 1-10, 2003.

[39] "FEMLAB User Guide and Introduction," ver. 2.2, Comsol AB, Stockholm, Sweden, 2001.

[40] D. J. Murray-Smith, "Advances in simulation model validation: theory, software and applications," in Proc. EUROSIM'95, 1995, pp. 75-84.

[41] L. A. Geddes and L. E. Baker, "The specific resistance of biological material-A compendium of data for the biomedical engineer and physiologist," Med. Biol. Eng., vol. 5, pp. 271-293, 1967.

[42] D. C. Barber and B. H. Brown, "Applied potential tomography," $J$. Phys. E.: Sci. Intsrum., vol. 17, pp. 723-733, 1984

[43] H. P. Schwan, "Electric characteristics of tissue," Biophysik, vol. 1, pp. 198-208, 1963.

[44] A. J. Surowiec, S. Stuchly, J. R. Barr, and A. Swarp, "Dielectric properties of breast carcinoma and the surrounding tissues," IEEE Trans. Biomed. Eng., vol. 35, no. 4, pp. 257-263, Apr. 1988.

[45] T. J. William, Y. Zhang, C. Li, and R. L. Jirtle, "The measured properties of normal and malignant human tissues from 50 to $900 \mathrm{MHz}$," Med. Phys, vol. 21, pp. 4-7, 1994.
[46] S. R. Smith, K. R. Foster, and G. L. Wolf, "Dielectric properties of VX-2 carcinoma versus normal liver tissue," IEEE Trans. Biomed. Eng., vol. BME-33, no. 5, pp. 522-524, May 1986.

[47] M. Pavlin, M. Kandušer, M. Reberšek, G. Pucihar, F. X. Hart, R. Magjarević, and D. Miklavčič, "Effect of cell electroporation on the conductivity of a cell suspension," Biophys. J., vol. 88, pp. 4378-4390, 2005.

[48] R. Fletcher and S. Leyffer, "Solving mixed integer nonlinear programs by outer approximation," Math. Programming, vol. 66, pp. 327-349, 1994.

[49] R. J. Dakin, "A tree-search algorithm for mixed integer programming problems," Comput. J., vol. 8, pp. 250-255, 1965.

[50] R. A. Gilbert, M. J. Jaroszeski, and R. Heller, "Novel electrode designs for electrochemotherapy," Biochimica et Biophysica Acta., vol. 1334, no. 1, pp. 9-14, 1997.

[51] M. Pavlin and D. Miklavčič, "Conductivity of a suspension of permeabilised cells_A theoretical analysis," Biophys. J., vol. 85, 2003.

[52] R. Davalos, B. Rubinsky, and D. M. Otten, "A feasibility study for electrical impedance tomography as a means to monitor tissue electroporation for molecular medicine," IEEE Trans. Biomed. Eng., vol. 49, no. 4, pp. 400-403, Apr. 2002.

[53] R. V. Davalos, L. M. Mir, and B. Rubinsky, "Tissue ablation with irreversible electroporation," Ann. Biomed. Eng., vol. 33, pp. 223-231, 2005.

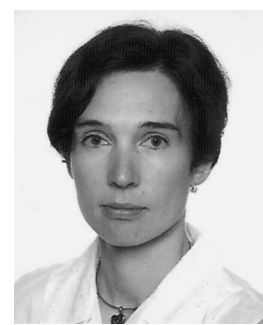

Davorka Šel was born in 1970. She received the M.Sc. and Ph.D. degrees in electrical engineering from the University of Ljubljana, Ljubljana, Slovenia.

Currently she is an Associate Researcher at the University of Ljubljana. Her main research interest is in the field of electropermeabilization, numerical modeling of electric field distribution in tissue during electropermeabilization, and optimization of electropermeabilization parameters.

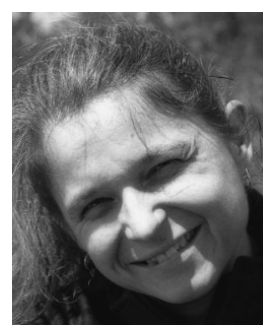

Alenka Maček Lebar received the M.Sc. and Ph.D. degrees in electrical engineering from the University of Ljubljana, Ljubljana, Slovenia, in 1995 and 1999, respectively.

She is an Assistant Professor with the Faculty of Electrical Engineering, University of Ljubljana. Her main research is directed toward the biomedical engineering field, including numerical modeling and electroporation-assisted drug delivery.

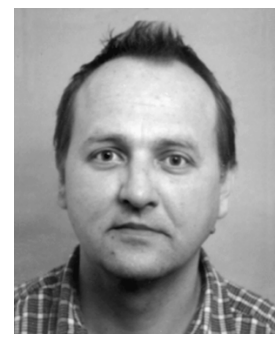

Damijan Miklavčič was born in 1963 in Ljubljana, Slovenia. He received the Ph.D. degree in electrical engineering from the University of Ljubljana.

$\mathrm{He}$ is Professor at the University of Ljubljana, with the Faculty of Electrical Engineering, and the Head of Laboratory of Biocybernetics. He is active in the field of biomedical engineering. His interest in the last years focuses on electroporation-assisted drug and gene delivery, including cancer treatment by means of electrochemotherapy, tissue oxygenation, modeling of biological processes, and hardware development. 